\title{
A robust study on 2019-nCOV outbreaks through non-singular derivative
}

\author{
Muhammad Altaf Khan ${ }^{1,2, a}$, Saif Ullah ${ }^{3}$, Sunil Kumar ${ }^{4, b}$ \\ ${ }_{1}^{1}$ Informetrics Research Group, Ton Duc Thang University, Ho Chi Minh City, Vietnam \\ 2 Faculty of Mathematics and Statistics, Ton Duc Thang University, Ho Chi Minh City, Vietnam \\ 3 Department of Mathematics, University of Peshawar, Peshawar, Pakistan \\ ${ }^{4}$ Department of Mathematics, National Institute of Technology, Jamshedpur, Jharkhand, India
}

Received: 28 December 2020 / Accepted: 27 January 2021

(C) The Author(s), under exclusive licence to Società Italiana di Fisica and Springer-Verlag GmbH Germany, part of Springer Nature 2021

\begin{abstract}
The new coronavirus disease is still a major panic for people all over the world. The world is grappling with the second wave of this new pandemic. Different approaches are taken into consideration to tackle this deadly disease. These approaches were suggested in the form of modeling, analysis of the data, controlling the disease spread and clinical perspectives. In all these suggested approaches, the main aim was to eliminate or decrease the infection of the coronavirus from the community. Here, in this paper, we focus on developing a new mathematical model to understand its dynamics and possible control. We formulate the model first in the integer order and then use the Atangana-Baleanu derivative concept with a nonsingular kernel for its generalization. We present some of the necessary mathematical aspects of the fractional model. We use a nonlinear fractional Lyapunov function in order to present the global asymptotical stability of the model at the disease-free equilibrium. In order to solve the model numerically in the fractional case, we use an efficient modified Adams-Bashforth scheme. The resulting iterative scheme is then used to demonstrate the detailed simulation results of the $\mathrm{ABC}$ mathematical model to examine the importance of the memory index and model parameters on the transmission and control of COVID-19 infection.
\end{abstract}

\section{Introduction}

The novel coronavirus disease is a viral infection caused by a newly discovered virus named as a coronavirus (SARS-CoV-2). This virus belongs to the family of viruses known as Coronaviridae which makes the causes of COVID-19. The first outbreak of COVID-19 infection is reported in Wuhan China at the end of 2019. This virus is highly contagious and due to its fast spreading mostly the faced it in the form of infected cases, deaths and economic loss [1]. The pandemic not only affects the humans population but also gives big loss to the countries' economic growth. A lot of people lost their jobs, and many industries and other business hubs suffered a lot. The recent report of the World Health Organization (WHO) reveals that a total of 10.27 million are tested positive and more than 1.52 million deaths

\footnotetext{
a e-mail: muhammad.altaf.khan@tdtu.edu.vn

b e-mail: skumar.math@nitjsr.ac.in (corresponding author)
} 
have been reported till December 12, 2020 [2]. It can also be noted that the recovery rate of this infection is greater than the mortality rate as 42.2 million of the confirmed infected cases are completely recovered so far. Currently, there is no available licensed medication or vaccine that protects or cures COVID-19. The medication given to infected people is mainly supportive treatment that aims at reducing symptoms. A person infected with the coronavirus may experience some of the common symptoms such as fever, fatigue, dry cough, shortness of breath or shortness of breath. Less common symptoms include pain, chills, diarrhea, sore throat, conjunctivitis, headache, skin eruptions, loss of taste or odor, discoloration, etc. [3,4]. The main intervention used for the minimization or eradication of COVID-19 infection in world is non-pharmaceutical, i.e., stay at home, wear a mask, social distance, avoid infected surfaces, frequent self-isolation, hand washing or sanitizing, lockdown, etc. Still the transmission routes of this novel infectious disease are not identified, but the main routes of the virus causing the COVID-19 infection are through droplets that can be produced during the coughs, sneezes or exhales of infectious person. These droplets are much heavy to hang in the air and then fall on surfaces or floors. The virus can be transferred to a person by touching the contaminated surfaces and then his/her eyes, nose or mouth.

Mathematical models can be used as an efficient tool to explore the dynamics of transmission and, ultimately, to suggest appropriate strategies to eradicate or minimize an infectious disease [5-12]. Recently, due to the COVID-19 pandemic, one of the challenges is that without any specific treatment how to minimize the incidence of this disease with nonpharmaceutical intervention in the whole world. Since the beginning of infection, a lot of mathematical models addressing the corona virus infection in different parts of the world are published. Most of the suggested mathematical models in this regard are formulated with the help of ordinary, partial or stochastic differential equations. For instant, the impact and role of non-pharmaceutical control measures on the COVID-19 incidence are studied in [13]. The authors in [13] further extend the work by providing optimal control strategies against COVID-19. A similar study was conducted in [14]. Additionally, in [14] the authors considered the role of environmental viral load on the COVID-19 dynamics. The global stability and the cost-effective analysis of a novel COVID-19 transmission model coupled with real data of Ghana have been carried out in [15]. The numerical simulations of an SEIR COVID19 epidemic model utilizing the real data from Indonesia were presented in [16] and others $[17,18]$.

The mathematical modeling approach via the fractional differential equation is another helping tool and gaining much interest in the recent years from the researchers point of view. Due to some of the interesting properties of the fractional models such as the memory, the heredity properties make it more powerful than the ordinary order mathematical models. It is due to the fact that the fractional epidemic models have the ability by capturing the fading memory and the crossover behavior that exhibits by the biological process. Moreover, the most interesting is the data fitting that can be considered better with fractional order rather than ordinary order, see, for more explanations, $[19,20]$. Some fractional operators that are classified on its kernel as a singular or non-singular kernels are used effectively in modeling of real-world problems, see [21,22]. The fractional operator introduced in [21] commonly known as the Atangana-Baleanu-Caputo $(\mathrm{ABC})$ derivative having its foundations on the Mittag-Leffler kernel, and it is non-singular and non-local. Therefore, mathematical models formulated via the $\mathrm{ABC}$ operator are more prominent and better explore the dynamics of a disease. Presently, in the literature, many researchers formulated mathematical models in fractional derivatives suggested as a best tool for the real-life problem solution. For example, in [23] the authors studied the dynamical behavior of COVID-19 infection in the Wuhan China with the help of fractional mathematical model based on $\mathrm{ABC}$ derivative. A similar 
study was conducted in [24], in which the authors analyzed the dynamical analysis of the corona infection through fractional model studied using the exponential type kernel. A novel coronavirus model has been recently introduced in [25] using the Caputo derivative. Recently, in [26], the authors have presented a numerical investigation of fractional COVID-19 model in fractional derivative. More recently, the authors have analyzed the dynamics of corona virus infection through non-integer-order model in [27]. The dynamical analysis of the coronavirus model considering KSA real data is studied in [28].

Keeping the above facts in mind, in the current study, we reformulate the COVID-19 model [13] by applying the $\mathrm{ABC}$ fractional-order operator. We will analyze the model dynamics with these new operators and its appropriateness to our proposed model. In order to formulate the fractional COVID-19 model, we replace the ordinary derivative with the generalized ABC fractional derivative. Moreover, we carried out a detailed theoretical and numerical aspects of the proposed model in fractional derivative. Organizing the reaming work is as follows: Sect. 2 presents the basics definitions relevant to the fractional-order derivatives that will be used in the modeling considered. The model formulation in $\mathrm{ABC}$ fractional derivative is discussed in Sect. 3. Section 4 presents some necessary mathematical properties of the proposed model. The numerical solution and the resulting simulation results for memory index and various parameters are shown in Sects. 5 and 6, respectively. The concluding remarks relevant to the present investigation are summarized in Sect. 7.

\section{Preliminary results}

This section presents some important necessary concepts and basic definition regarding fractional operators.

Definition 2.1 For the function denoted by $y(t) \in C^{n}$, the Caputo type derivative having order $\vartheta$ in $(n-1, n]$ where $n \in \mathbb{N}$ is defined as [21]:

$$
{ }^{C} D_{t}^{\vartheta}(y(t))=\frac{1}{\Gamma(n-\vartheta)} \int_{0}^{t} \frac{y^{n}(\zeta)}{(t-\zeta)^{\vartheta-n+1}} \mathrm{~d} \zeta .
$$

Clearly ${ }^{C} D_{t}^{\vartheta}(y(t))$ approaches $y^{\prime}(t)$ as $\vartheta \rightarrow 1$.

Definition 2.2 The ABC fractional operator is defined as follows: [21]:

$$
{ }_{a}^{\mathrm{ABC}} D_{t}^{\vartheta} y(t)=\frac{A B(\vartheta)}{1-\vartheta} \int_{a}^{t} y^{\prime}(x) E_{\vartheta}\left[\frac{-\vartheta(t-x)^{\vartheta}}{1-\vartheta}\right] \mathrm{d} x,
$$

where $0 \leq \vartheta \leq 1, f \in C[a, b]$ and $A B(\vartheta)$ is the normalized function and $A B(0)=$ $A B(1)=1$.

Definition 2.3 The associated integral to the fractional derivative with Mittag-Leffler kernel is defined as follows [21]:

$$
\left.{ }_{0}^{\mathrm{ABC}} I_{t}^{\vartheta} y(t)=\frac{1-\vartheta}{A B(\vartheta)} y(t)+\frac{\vartheta}{A B(\vartheta) \Gamma(\vartheta)} \int_{0}^{t} y(x)(t-x)^{\vartheta-1}\right] \mathrm{d} x .
$$

Consider the system of fractional differential equation in Atangana-Baleanu-Caputo sense described as follows [29]:

$$
\left(\begin{array}{l}
\mathrm{ABC} \\
t_{0}
\end{array} D_{t}^{\vartheta} y\right)(t)=\mathrm{F}(t, y),
$$

where $\mathrm{F}: \mathrm{D} \subset \mathbb{R}^{n} \times \mathbb{R}_{+} \longrightarrow \mathbb{R}^{n}$ and $\vartheta \in(0,1]$. Then, the following result deals with the globally asymptotic stability of fractional system (4). 
Theorem 2.1 [29] For the fractional system (4) with Atangana-Baleanu-Caputo derivative, let $\mathcal{V}(t, y(t))$ be positive definite and $\mathcal{V}(t)=\mathcal{V}(t, y(t))$ be a continuously differentiable function. Further, ${ }_{t_{0}}^{\mathrm{ABC}} D_{t}^{\vartheta} \mathcal{V}(t, y(t))$ is negative definite for all $\vartheta \in(0,1]$. If there exists a function say $F$ of class $K$ with $\mathcal{V}(y)=F(y)$ such that if $\mathcal{V}$ increase, then $F$ increases, then the equilibrium point $y=0$ is asymptotically stable point at $t=0$.

\section{The COVID-19 compartmental model}

Initially, we present brief details of the classical integer-order compartmental model describing the dynamics of novel COVID-19 infection. The model under consideration has been recently studied in [13]. The transmission model is formulated by sub-dividing the total population described by $N(t)$ at time $t$, into mutually exclusive eight sub-population groups. The respective sub-groups are the susceptible humans not yet infected but at risk to catch the infection $S(t)$, exposed to COVID-19 infection $E(t)$, the symptomatic humans are those infected individuals having clinical symptoms $I(t)$, the asymptomatic infected humans are those having no or mild clinical symptoms of COVID-19 $I_{A}(t)$, the quarantined humans $Q(t)$, the hospitalized (or in self-isolation) humans $I_{H}(t)$, the COVID-19 infected humans who are in critical situation $I_{C}(t)$ and those individuals that are recovered from COVID-19 infection are describing through the recovered class, $R(t)$. In the considered model, it is considered that the hospitalized class $I_{H}(t)$ can transmit the infection further. The biological description and respective numerical values of the parameters involved in the model are described in Table (1). The resulting COVID-19 mathematical model is formulated with the help of the following nonlinear system of classical differential equations:

$$
\left\{\begin{array}{l}
\frac{\mathrm{d} S}{\mathrm{~d} t}=\Delta-\frac{\beta\left(I+\beta_{A} I_{A}+\beta_{H} I_{H}\right)}{N} S-\mathrm{d} S, \\
\frac{\mathrm{d} E}{\mathrm{~d} t}=\frac{\beta\left(I+\beta_{A} I_{A}+\beta_{H} I_{H}\right)}{N} S-(\kappa+\delta+d) E, \\
\frac{\mathrm{d} I}{\mathrm{~d} t}=r \kappa E-\left(r_{1}+d+d_{1}+\gamma\right) I, \\
\frac{\mathrm{d} I_{A}}{\mathrm{~d} t}=(1-r) \kappa E-\left(r_{2}+d\right) I_{A}, \\
\frac{\mathrm{d} Q}{\mathrm{~d} t}=\delta E-\left(\mu+r_{3}+\zeta\right) Q, \\
\frac{\mathrm{d} I_{H}}{\mathrm{~d} t}=\gamma I+\zeta Q-\left(d+r_{4}+d_{2}+\phi\right) I_{H}, \\
\frac{\mathrm{d} I_{C}}{\mathrm{~d} t}=\phi I_{H}-\left(d+r_{5}+d_{3}\right) I_{C}, \\
\frac{\mathrm{d} R}{\mathrm{~d} t}=r_{1} I+r_{2} I_{A}+r_{3} Q+r_{4} I_{H}+r_{5} I_{C}-\mathrm{d} R,
\end{array}\right.
$$

subjected to initial conditions (ICs):

$$
\left\{\begin{array}{l}
S(0)=S_{0} \geq 0, \quad E(0)=E_{0} \geq 0, \quad I(0)=I_{0} \geq 0, \quad I_{A}(0)=I_{A 0} \geq 0 \\
Q(0)=Q_{0} \geq 0, \quad I_{H}(0)=I_{H 0} \geq 0, \quad I_{C}(0)=I_{C 0} \geq 0, \quad R(0)=R_{0} \geq 0 .
\end{array}\right.
$$


3.1 The COVID-19 model derivation using the non-singular operator

The fractional derivative in $\mathrm{ABC}$ sense is more generalized as the kernel involved in this operator is both non-local and non-singular. Moreover, this operator has the ability by capturing the memory effect and the crossover behavior exists in the processes of biology models. Keeping these facts in mind, in this section, we reformulate the COVID-19 mathematical model (5) with the help of non-integer operator in $\mathrm{ABC}$ case with the order $\vartheta \in(0,1]$. The resulting non-integer-order mathematical model shown below is obtained with the replacement of the ordinary derivative by the fractional-order $\mathrm{ABC}$ operator:

$$
\left\{\begin{array}{l}
{ }^{A B C} D_{t}^{\vartheta} S(t)=\Delta-\frac{\beta\left(I+\beta_{A} I_{A}+\beta_{H} I_{H}\right)}{N} S-\mathrm{d} S, \\
{ }^{A B C} D_{t}^{\vartheta} E(t)=\frac{\beta\left(I+\beta_{A} I_{A}+\beta_{H} I_{H}\right)}{N} S-(\kappa+\delta+d) E, \\
{ }^{A B C} D_{t}^{\vartheta} I(t)=r \kappa E-\left(r_{1}+d+d_{1}+\gamma\right) I, \\
A B C D_{t}^{\vartheta} I_{A}(t)=(1-r) \kappa E-\left(r_{2}+d\right) I_{A}, \\
{ }^{A B C} D_{t}^{\vartheta} Q(t)=\delta E-\left(d+r_{3}+\zeta\right) Q, \\
{ }^{A B C} D_{t}^{\vartheta} I_{H}(t)=\gamma I+\zeta Q-\left(d+r_{4}+d_{2}+\phi\right) I_{H}, \\
{ }^{A B C} D_{t}^{\vartheta} I_{C}(t)=\phi I_{H}-\left(d+r_{5}+d_{3}\right) I_{C}, \\
A B C D_{t}^{\vartheta} R(t)=r_{1} I+r_{2} I_{A}+r_{3} Q+r_{4} I_{H}+r_{5} I_{C}-\mathrm{d} R,
\end{array}\right.
$$

subjected to the nonnegative ICs (6). For simplicity, let us take

$$
\lambda(t)=\frac{\beta\left(I+\beta_{A} I_{A}+\beta_{H} I_{H}\right)}{N},
$$

and

$$
\begin{aligned}
& \mathrm{m}_{1}=(\kappa+\delta+d), \quad \mathrm{m}_{2}=\left(r_{1}+d+d_{1}+\gamma\right), \quad \mathrm{m}_{3}=\left(r_{2}+d\right), \quad \mathrm{m}_{4}=\left(d+r_{3}+\zeta\right), \\
& \mathrm{m}_{5}=\left(d+r_{4}+d_{2}+\phi\right), \quad \mathrm{m}_{6}=\left(d+r_{5}+d_{3}\right) .
\end{aligned}
$$

With the above suggestions, we can write the model in the following form:

$$
\left\{\begin{array}{l}
{ }^{A B C} D_{t}^{\vartheta} S(t)=\Lambda-\lambda S-\mathrm{d} S, \\
{ }^{A B C} D_{t}^{\vartheta} E(t)=\lambda S-\mathrm{m}_{1} E, \\
{ }^{A B C} D_{t}^{\vartheta} I(t)=r \kappa E-\mathrm{m}_{2} I, \\
{ }^{A B C} D_{t}^{\vartheta} I_{A}(t)=(1-r) \kappa E-\mathrm{m}_{3} I_{A}, \\
{ }^{A B C} D_{t}^{\vartheta} Q(t)=\delta E-\mathrm{m}_{4} Q, \\
{ }^{A B C} D_{t}^{\vartheta} I_{H}(t)=\gamma I+\zeta Q-\mathrm{m}_{5} I_{H}, \\
{ }^{A B C} D_{t}^{\vartheta} I_{C}(t)=\phi I_{H}-\mathrm{m}_{6} I_{C}, \\
{ }^{A B C} D_{t}^{\vartheta} R(t)=r_{1} I+r_{2} I_{A}+r_{3} Q+r_{4} I_{H}+r_{5} I_{C}-\mathrm{d} R .
\end{array}\right.
$$

In the above COVID-19 compartmental model (8), the ${ }^{A B C} D_{t}^{\vartheta}$ denotes the fractional operator in $\mathrm{ABC}$ sense.

Parameter estimations

The estimation of model parameters is essential and realistic approach to predict and explore the dynamics of a disease. In this part of the paper, we utilized the well-known least square 
Table 1 Models parameters and its estimated values

\begin{tabular}{llll}
\hline Parameter & Description & Value/day & Source \\
\hline$\Delta$ & Recruitment rate & $\mu * N(0)$ & Estimated \\
$d$ & Death rate naturally & $\frac{1}{365 \times 67.7)}$ & {$[2]$} \\
$d_{1}$ & The death rate due to symptomatic infection & 0.0100 & Fitted \\
$\beta_{A}$ & Transmissible coefficient rate related to $I_{A}$ & 0.5932 & Fitted \\
$\kappa$ & Incubation period & 0.3233 & Fitted \\
$r$ & Proportion of the symptomatic infection & 0.4760 & Fitted \\
$\gamma$ & Hospitalized rate for the symptomatic people & 0.3738 & Fitted \\
$d_{2}$ & Death of the people in the hospitalization class & 0.0131 & Fitted \\
$d_{3}$ & Death of the people critical-infected class & 0.039 & Fitted \\
$r_{1}$ & Recovery due to $I$ & 0.4368 & Fitted \\
$r_{2}$ & Recovery due to $I_{A}$ & 0.2550 & Fitted \\
$r_{3}$ & Rate of recover of quarantined individuals & 0.2562 & Fitted \\
$r_{4}$ & Rate of recover of hospitalized individuals & 0.1010 & Fitted \\
$r_{5}$ & Rate of recover of critically infected individuals & 0.0261 & Fitted \\
$\delta$ & Rate of quarantined for exposed individuals & 0.4818 & Fitted \\
$\beta$ & Transmission coefficient that generate the infection & 0.6349 & Fitted \\
$\zeta$ & The rate by which the quarantined are hospitalized & 0.5435 & Fitted \\
$\beta_{H}$ & Transmissible coefficient relative to $I_{H}$ & 0.6312 & Fitted \\
$\phi$ & The individuals movement from $I_{H}$ to $I_{C}$ & 0.1950 & Fitted \\
\hline
\end{tabular}

curve fitting statistical technique in order to get the realistic parameters values for the confirmed COVID-19 infected cases reported in Pakistan from March 1 to 31 August 2020. Some of the demographic parameters such as the birth $\Delta$ and natural mortality rate $d$ are estimated through literature and given in Table 1 . The reasonable fitted model predicted curve is depicted in Fig. 1. The updated estimated value of the basic reproductive number evaluated via the fitted parameters is $\mathcal{R}_{0} \approx 1.32$. The initial values of model variables are assumed as $S(0)=220870336, E(0)=20000, I(0)=4, I_{A}(0)=3000, Q(0)=0, I_{H}(0)=0$, $I_{C}(0)=0$, and $R(0)=0$.

\section{Basic properties of the ABC COVID-19 model}

The following feasible region is well posed and invariant for the ABC fractional model (8), where its dynamics can be analyzed:

$$
\Omega \subset \mathbb{R}_{+}^{8},
$$

such that

$$
\begin{aligned}
\Xi= & \left\{\left(S(t), E(t), I(t), I_{A}(t), Q(t), I_{H}(t), I_{C}(t), R(t)\right) \in \mathbb{R}_{+}^{8}: S(t)+E(t)\right. \\
& \left.+I(t)+I_{A}(t)+Q(t)+I_{H}(t)+I_{C}(t)+R(t) \leq \Delta / d\right\} .
\end{aligned}
$$




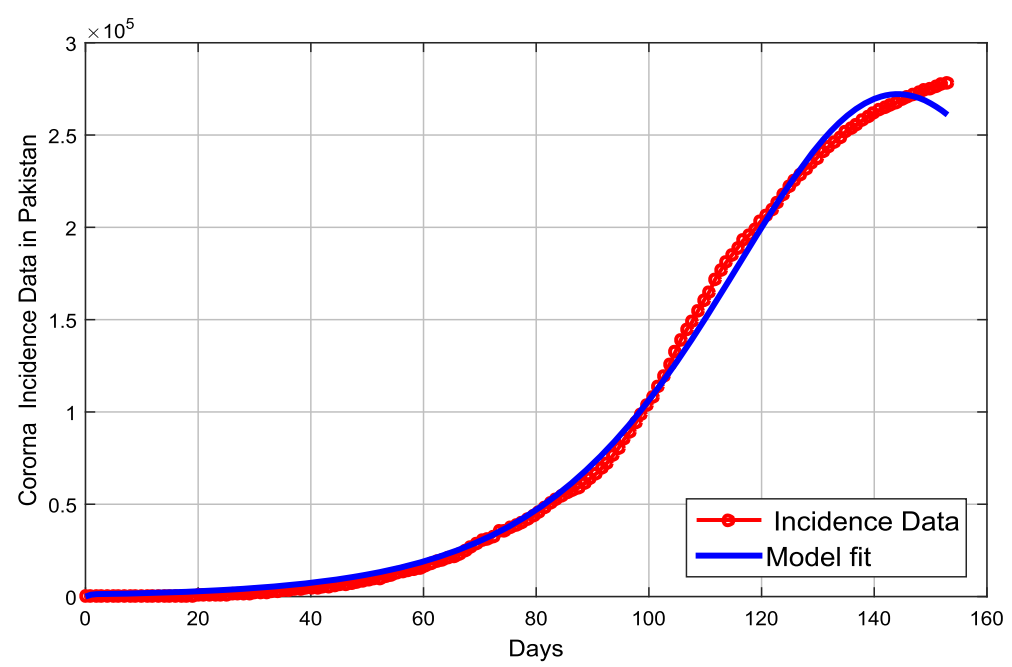

Fig. 1 Comparison of model with real cases: circle denotes real cases, while bold line is the model solution. The data considered here from March 1 to August 31, 2020

The dynamics of the total population is obtained by adding all equations of the model (8);

$$
\begin{aligned}
{ }^{A B C} D_{t}^{\vartheta} N(t)= & { }^{A B C} D_{t}^{\vartheta} S(t)+{ }^{A B C} D_{t}^{\vartheta} E(t)+{ }^{A B C} D_{t}^{\vartheta} I(t)+{ }^{A B C} D_{t}^{\vartheta} I_{A}(t)+{ }^{A B C} D_{t}^{\vartheta} Q(t) \\
& +{ }^{A B C} D_{t}^{\vartheta} I_{H}(t)+{ }^{A B C} D_{t}^{\vartheta} I_{C}(t)+{ }^{A B C} D_{t}^{\vartheta} R(t) .
\end{aligned}
$$

Hence;

$$
{ }^{A B C} D_{t}^{\vartheta} N(t)=\Delta-d N(t)-d_{1} I(t)-d_{2} I_{H}(t)-d_{3} I_{C}(t) \leq \Delta-\mathrm{d} N(t) .
$$

We have then,

$$
{ }^{A B C} D_{t}^{\vartheta} N(t)+\mathrm{d} N(t) \leq \Delta .
$$

The application of Laplace transform gives the following:

$$
\lim _{t \rightarrow \infty} N(t) \leq \Delta / d
$$

Thus, $N(t)$ approaches $\Delta / d$ whenever $t$ approaches $\infty$ and $\forall t>0$, all the solutions possessing by the system with its appropriate initial values of the model variables in $\Xi$ will remain in $\Xi$. So, the region $\Xi$ is said to be positive invariant and will attract all the solutions in $\mathbb{R}_{+}^{8}$.

\subsection{Equilibria of the ABC COVID-19 model}

The obtaining of the equilibrium points is the important concept in dynamical system, by which the stability of the system can be analyzed through that equilibrium point. Typically, in most of the biological models formulated for humans dynamics possess two equilibrium points, that is, the disease-free and the endemic. In order to get the disease-free equilibrium $\mathrm{DFE}$ of the model (8), we show it by

$$
\mathcal{D}_{0}^{* *}=\left(S^{0}, E^{0}, I^{0}, I_{A}^{0}, Q^{0}, I_{H}^{0}, I_{C}^{0}, R^{0}\right),
$$


and obtained is as follows:

$$
\mathcal{D}_{0}^{* *}=\left(\frac{\Delta}{d}, 0,0,0,0,0,0,0\right)
$$

Another important concept in the disease epidemiology is the computation of the basic reproduction number. It is also called a threshold quantity that characterized the disease whether it can be controlled or not. The value of the basic production number less or greater decides the disease-free and endemicity, respectively, in the population. There are many methods to compute the basic reproduction for the biological models but the one that using the researchers mostly in their work is the next-generation technique [30]. Commonly, the basic reproduction number is denoted by $\mathcal{R}_{0}$. So, by using the method, by letting, $x=$ $\left(E, I, I_{A}, Q, I_{H}, I_{C}\right)^{T}$, then, we have

$$
\frac{\mathrm{d} x}{\mathrm{~d} t}=\mathcal{F}-\mathcal{V}
$$

where

$$
\mathcal{F}=\left(\begin{array}{c}
\frac{\beta\left(I+\beta_{A} I_{A}+\beta_{H} I_{H}\right) S}{N} \\
0 \\
0 \\
0 \\
0 \\
0
\end{array}\right), \quad \mathcal{V}=\left(\begin{array}{c}
\mathrm{m}_{1} E \\
\mathrm{~m}_{2} I-r \kappa E \\
\mathrm{~m}_{3} I_{A}-(1-r) \kappa I_{A} \\
\mathrm{~m}_{4} Q-\delta E \\
\mathrm{~m}_{5} I_{H}-\gamma I-\zeta Q \\
\mathrm{~m}_{6} I_{C}-\phi I_{H}
\end{array}\right) .
$$

The basic reproduction number $\mathcal{R}_{0}$ at the DFE can be achieved using $\rho\left(\mathbf{F V}^{-1}\right)$ for our considered model (8):

$$
\mathcal{R}_{0}=\frac{\beta\left[\mathrm{m}_{2} \mathrm{~m}_{3} \delta \zeta \beta_{H}+\mathrm{m}_{4} \kappa\left(\mathrm{m}_{3} r\left(\mathrm{~m}_{5}+\beta_{H} \gamma\right)+\mathrm{m}_{2} \mathrm{~m}_{5} \beta_{A}(1-r)\right)\right]}{\mathrm{m}_{1} \mathrm{~m}_{2} \mathrm{~m}_{3} \mathrm{~m}_{4} \mathrm{~m}_{5}} .
$$

The stability of the model will be analyzed through the basic reproduction number $\mathcal{R}_{0}$.

\subsection{Lyapunov stability of DFE case}

We present here the global dynamics of the fractional model (8) at the $\mathrm{DFE} \mathcal{D}_{0}^{* *}$ by constructing the Lyapunov function.

Theorem 4.1 If $\mathcal{R}_{0}<1$, then the model (8) at $\mathcal{D}_{0}^{* *}$ is globally asymptotically stable.

Proof We take the following suitable Lyapunov function in consideration to prove the desired result:

$$
\mathfrak{L}(t)=\left(\frac{\mathcal{R}_{0} \mathrm{~m}_{5}}{\beta \beta_{H}}\right) E+\left(\frac{\mathrm{m}_{5}+\beta_{H} \gamma}{\mathrm{m}_{2} \beta_{H}}\right) I+\left(\frac{\mathrm{m}_{5} \beta_{A}}{\mathrm{~m}_{3} \beta_{H}}\right) I_{A}+\left(\frac{\zeta}{\mathrm{m}_{4}}\right) Q+I_{H} .
$$


The time fractional derivative of $\mathfrak{L}(t)$ in $\mathrm{ABC}$ sense along the solution of model (8) is as follows:

$$
\begin{aligned}
& { }^{A B C} D_{t}^{\vartheta} \mathfrak{L}(t)=\left(\frac{\mathcal{R}_{0} \mathrm{~m}_{5}}{\beta \beta_{H}}\right)^{A B C} D_{t}^{\vartheta} E+\left(\frac{\mathrm{m}_{5}+\beta_{H} \gamma}{\mathrm{m}_{2} \beta_{H}}\right)^{A B C} D_{t}^{\vartheta} I+\left(\frac{\mathrm{m}_{5} \beta_{A}}{\mathrm{~m}_{3} \beta_{H}}\right)^{A B C} D_{t}^{\vartheta} I_{a} \\
& +\left(\frac{\zeta}{\mathrm{m}_{4}}\right){ }^{A B C} D_{t}^{\vartheta} Q+{ }^{A B C} D_{t}^{\vartheta} I_{H}, \\
& =\frac{\mathcal{R}_{0} \mathrm{~m}_{5}}{\beta \beta_{H}}\left[\frac{\beta S\left(I+\beta_{A} I_{A}+\beta_{H} I_{H}\right)}{N}-\mathrm{m}_{1} E\right]+\left(\frac{\mathrm{m}_{5}+\beta_{H} \gamma}{\mathrm{m}_{2} \beta_{H}}\right)\left(r \kappa E-\mathrm{m}_{2} I\right) \\
& +\left(\frac{\mathrm{m}_{5} \beta_{A}}{\mathrm{~m}_{3} \beta_{H}}\right)\left((1-r) \kappa E-\mathrm{m}_{3} I_{A}\right)+\left(\frac{\zeta}{\mathrm{m}_{4}}\right)\left(\delta E-\mathrm{m}_{4} Q\right)+\gamma I \\
& +\zeta Q-\mathrm{m}_{5} I_{H}, \\
& \leq \frac{\mathcal{R}_{0} \mathrm{~m}_{5}}{\beta \beta_{H}}\left[\beta\left(I+\beta_{A} I_{A}+\beta_{H} I_{H}\right)-\mathrm{m}_{1} E\right]+\left(\frac{\mathrm{m}_{5}+\beta_{H} \gamma}{\mathrm{m}_{2} \beta_{H}}\right)\left(r \kappa E-\mathrm{m}_{2} I\right) \\
& +\left(\frac{\mathrm{m}_{5} \beta_{A}}{\mathrm{~m}_{3} \beta_{H}}\right)\left((1-r) \kappa E-\mathrm{m}_{3} I_{A}\right)+\left(\frac{\zeta}{\mathrm{m}_{4}}\right)\left(\delta E-\mathrm{m}_{4} Q\right)+\gamma I \\
& +\zeta Q-\mathrm{m}_{5} I_{H}, \quad S / N \leq 1 \text {. } \\
& =\left(\frac{\mathcal{R}_{0} \mathrm{~m}_{5}}{\beta_{H}} I-\frac{\mathrm{m}_{5}}{\beta_{H}} I\right)+\left(\frac{\mathcal{R}_{0} \mathrm{~m}_{5}}{\beta_{H}} \beta_{A} I_{A}-\frac{\mathrm{m}_{5}}{\beta_{H}} \beta_{A} I_{A}\right)+\left(\mathcal{R}_{0} \mathrm{~m}_{5} I_{H}-\mathrm{m}_{5} I_{H}\right) \\
& +\left(\frac{\delta \zeta}{\mathrm{m}_{4}}-\frac{\mathcal{R}_{0} \mathrm{~m}_{5} \mathrm{~m}_{1}}{\beta \beta_{H}} E+\left(\frac{\mathrm{m}_{5}+\beta_{H} \gamma}{\mathrm{m}_{2} \beta_{H}}\right) r \kappa E+\frac{\beta_{A} \mathrm{~m}_{5}}{\mathrm{~m}_{3} \beta_{H}}(1-r) \kappa E\right), \\
& =\frac{\mathrm{m}_{5}}{\beta_{H}}\left(\mathcal{R}_{0}-1\right) I+\frac{\mathrm{m}_{5}}{\beta_{H}}\left(\mathcal{R}_{0}-1\right) \beta_{A} I_{A}+\mathrm{m}_{5}\left(\mathcal{R}_{0}-1\right) I_{H}, \\
& \leq \frac{\mathrm{m}_{5}}{\beta_{H}}\left(\mathcal{R}_{0}-1\right)\left(I+\beta_{A} I_{A}+\beta_{H} I_{H}\right), \\
& \leq 0 \text {. }
\end{aligned}
$$

It is clear that if $\mathcal{R}_{0}<1$, then ${ }^{A B C} D_{t}^{\vartheta} \mathcal{L}(t)$ is negative. Thus, following the results stated in Theorem 2.1, it is concluded that the DFE $\mathcal{D}_{0}^{* *}$ is GAS in $\Xi$.

\subsection{Existence and uniqueness of the solution}

In this part of the manuscript, we present the important feature of the ABC COVID-19 epidemic model known as the existence and uniqueness (EU) as described in (8). For the desired EU result, the fixed-point theory is utilized. Initially, the COVID-19 epidemic model (8) is reformulated via the following initial value problem:

$$
\left\{\begin{array}{l}
{ }^{A B C} D_{t}^{\vartheta} \mathrm{Y}(t)=\mathrm{G}(t, \mathrm{Y}(t)) \\
\mathrm{Y}(0)=\mathrm{y}_{0}, \quad 0<t<\mathcal{T}<\infty
\end{array}\right.
$$

In the above problem (11), the vector shown by $\mathrm{y}=\left(S, E, I, I_{A}, Q, I_{H}, I_{C}, R\right)$ denotes the corresponding state variables and the vector $y_{0}=\left(S(0), E(0), I(0), I_{A}(0)\right.$, $\left.Q(0), I_{H}(0), I_{C}(0), R(0)\right)$ shows the corresponding ICs. Furthermore, G describes a continuous vector function as given below: 


$$
\mathrm{G}=\left(\begin{array}{c}
\mathrm{G}_{1} \\
\mathrm{G}_{2} \\
\mathrm{G}_{3} \\
\mathrm{G}_{4} \\
\mathrm{G}_{5} \\
\mathrm{G}_{6} \\
\mathrm{G}_{7} \\
\mathrm{G}_{8}
\end{array}\right)=\left(\begin{array}{c}
\Lambda-\lambda S-\mathrm{d} S \\
\lambda S-\mathrm{m}_{1} E \\
r \kappa E-\mathrm{m}_{2} I \\
(1-r) \kappa E-\mathrm{m}_{3} I_{A} \\
\delta E-\mathrm{m}_{4} Q \\
\gamma I+\zeta Q-\mathrm{m}_{5} I_{H} \\
\phi I_{H}-\mathrm{m}_{6} I_{C} \\
r_{1} I+r_{2} I_{A}+r_{3} Q+r_{4} I_{H}+r_{5} I_{C}-\mathrm{d} R
\end{array}\right) .
$$

In addition, the function defined as above $\mathrm{G}$ fulfills the Lipschitz criteria shown as follows:

$$
\left\|\mathrm{G}\left(t, \mathrm{Y}_{1}(t)\right)-\mathrm{G}\left(t, \mathrm{Y}_{2}(t)\right)\right\| \leq \mathrm{D}\left\|\mathrm{Y}_{1}(t)-\mathrm{Y}_{2}(t)\right\|, \quad \mathrm{D}>0 .
$$

Further, the following theorem deals with the required EU result.

Theorem 4.2 A unique solution of the proposed COVID-19 epidemic ABC mathematical model (11) will exists if the condition describe below holds:

$$
\psi_{1}(\vartheta) D+\psi_{2}(\vartheta) \mathcal{T}_{\max }^{\vartheta} D<1 .
$$

where,

$$
\psi_{1}(\vartheta)=\frac{(1-\vartheta)}{A B C(\vartheta)}, \quad \text { and } \quad \psi_{2}(\vartheta)=\frac{\vartheta}{A B C(\vartheta) \Gamma(\vartheta)}
$$

Proof Taking the integral in ABC sense upon the problem (11), we leads to the following nonlinear Volterra integral equation:

$$
\mathrm{y}=\mathrm{v}_{0}+\psi_{1}(\vartheta) \mathrm{G}(t, \mathrm{y})+\psi_{2}(\vartheta) \int_{0}^{t}(t-\varsigma)^{\vartheta-1} \mathrm{G}(\varsigma, v(\varsigma)) \mathrm{d} \varsigma .
$$

To move further, we define $\mathcal{J}=(0, \mathcal{T})$ and the corresponding operator $\mathrm{G}: \mathrm{C}\left(\mathcal{J}, \mathbb{R}^{8}\right) \rightarrow$ $\mathrm{C}\left(\mathcal{J}, \mathbb{R}^{8}\right)$ stated as

$$
\mathrm{G}[\mathrm{Y}]=v_{0}+\psi_{1}(\vartheta) \mathrm{G}(t, \mathrm{Y})+\psi_{2}(\vartheta) \int_{0}^{t}(t-\varsigma)^{\vartheta-1} \mathrm{G}(\varsigma, v(\varsigma)) \mathrm{d} \varsigma .
$$

In a result, Eq. (14) takes the following form:

$$
\mathrm{Y}=\mathrm{G}[\mathrm{y}] \text {. }
$$

We denote the supremum norm upon $\mathcal{J}$ by $\|\cdot\|_{\mathcal{J}}$ defines as follows:

$$
\|\mathrm{y}\|_{\mathcal{J}}=\sup _{t \in \mathcal{J}}\|\mathrm{y}\|, \quad \mathrm{y} \in \mathrm{C} .
$$

$\mathrm{C}\left(\mathcal{J}, \mathbb{R}^{8}\right)$ along with the norm shown by $\|\cdot\|_{\mathcal{J}}$ constructed a Banach space. Moreover, the following inequality can be easily shown:

$$
\left\|\int_{0}^{t} \mathrm{G}(t, \varsigma) \mathrm{Y}(\varsigma) \mathrm{d} \varsigma\right\| \leq \mathcal{T}\|\mathrm{G}(t, \varsigma)\|_{\mathcal{J}}\left\|_{\mathrm{Y}}\right\|_{\mathcal{J}},
$$

with $\mathrm{Y} \in \mathrm{C}\left(\mathcal{J}, \mathbb{R}^{8}\right), \mathrm{G}(t, \varsigma) \in \mathrm{C}\left(\mathcal{J}^{2}, \mathbb{R}\right)$ such that

$$
\|\mathrm{G}(t, \varsigma)\|_{\mathcal{J}}=\sup _{t, \varsigma \in \mathcal{J}}|\mathrm{G}(t, \varsigma)| .
$$


Utilizing Eq. (16), we lead to the following result

$$
\begin{aligned}
\left\|\mathrm{G}\left[\mathrm{Y}_{1}(t)\right]-\mathrm{G}\left[\mathrm{Y}_{2}(t)\right]\right\|_{\mathcal{J}} \leq & \| \psi_{1}(\vartheta)\left(\mathrm{G}\left(t, \mathrm{Y}_{1}(t)\right)-\mathrm{G}\left(t, \mathrm{Y}_{2}(t)\right)\right)+\psi_{2}(\vartheta) \times \\
& \int_{0}^{t}(t-\varsigma)^{\vartheta-1}\left(\mathrm{G}\left(\varsigma, \mathrm{y}_{1}(\varsigma)\right)-\mathrm{G}\left(\varsigma, \mathrm{y}_{2}(\varsigma)\right)\right) \mathrm{d} \varsigma \|_{\mathcal{J}}
\end{aligned}
$$

Further, making use of Eqs. (12), (18) as well as the triangular inequality, Eq. (20) gets the following form:

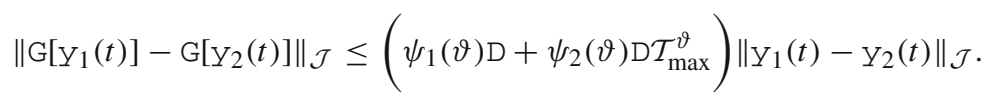

Finally, we obtain

$$
\left.\| \mathrm{G}\left[\mathrm{Y}_{1}(t)\right]-\mathrm{G}_{\mathrm{y}_{2}}(t)\right]\left\|_{\mathcal{J}} \leq \mathrm{M}\right\| \mathrm{Y}_{1}(t)-\mathrm{y}_{2}(t) \|_{\mathcal{J}},
$$

where,

$$
\mathrm{M}=\psi_{1}(\vartheta) \mathrm{D}+\psi_{2}(\vartheta) \mathrm{D} \mathcal{T}_{\max }^{\vartheta}
$$

The operator $\mathrm{G}$ will be a contraction if it holds the condition in Eq. (13). Hence, the system (11) has a unique solution.

\section{Numerical solution of fractional COVID-19 model}

In this section, we investigate the iterative scheme of the COVID-19 epidemic model (8) with $\mathrm{ABC}$ operator in order to present the graphical impact of various parameters and memory index on the disease incidence. An efficient numerical scheme based on the two-step Lagrange polynomial approximation [31] is used for this purpose. Firstly, a brief derivation of the iterative scheme is presented for the general Cauchy problem described in (11) and then it is implemented for the fractional COVID-19 model (8) in consideration.

Applying the integral operator in ABC sense as defined in (3) on both sides of Cauchy problem (11). In a result, we lead to the following integral equation:

$$
\mathrm{y}(t)-\mathrm{y}(0)=\psi_{1}(\vartheta) \mathrm{G}(t, \mathrm{y})+\psi_{2}(\vartheta) \int_{0}^{t} \mathrm{G}\left(x, \mathrm{y}(x)(t-x)^{\vartheta-1} \mathrm{~d} x,\right.
$$

where $\psi_{1}(\vartheta)$ and $\psi_{2}(\vartheta)$ are defined as in previous section. Further, at $t=t_{\mathrm{k}+1}=(\mathrm{k}+1) h$, we have

$$
\begin{aligned}
\mathrm{y}\left(t_{\mathrm{k}+1}\right)-\mathrm{y}(0)= & \psi_{1}(\vartheta) \mathrm{G}\left(t_{\mathrm{k}}, \mathrm{y}\left(t_{\mathrm{k}}\right)\right)+\psi_{2}(\vartheta) \int_{0}^{t_{\mathrm{k}+1}} \mathrm{G}(x, v(x))\left(t_{m+1}-x\right)^{\vartheta-1} \mathrm{~d} x, \\
= & \frac{1-\vartheta}{A B(\vartheta)} \mathrm{G}\left(t_{\mathrm{k}}, \mathrm{Y}\left(t_{\mathrm{k}}\right)\right) \\
& +\frac{\vartheta}{A B(\vartheta) \times \Gamma(\vartheta)} \sum_{j=0}^{\mathrm{k}} \int_{t_{j}}^{t_{j+1}} \mathrm{G}(x, \mathrm{Y}(x))\left(t_{\mathrm{k}+1}-x\right)^{\vartheta-1} \mathrm{~d} x .
\end{aligned}
$$

Now, with the help of polynomial interpolation approach, the vector function $\mathrm{G}(x, \mathrm{y}(x))$ is approximated using two-step Lagrange polynomial upon the interval $\left[t_{l}, t_{l+1}\right]$. Thus, we 


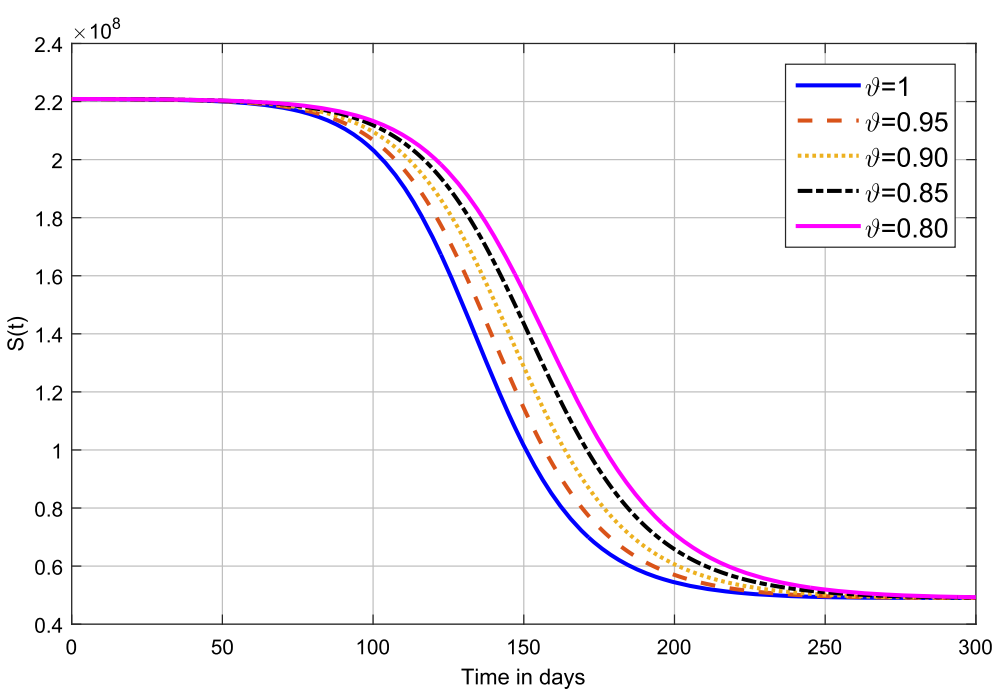

Fig. 2 Dynamics of suspectable individuals for various values of $\vartheta$

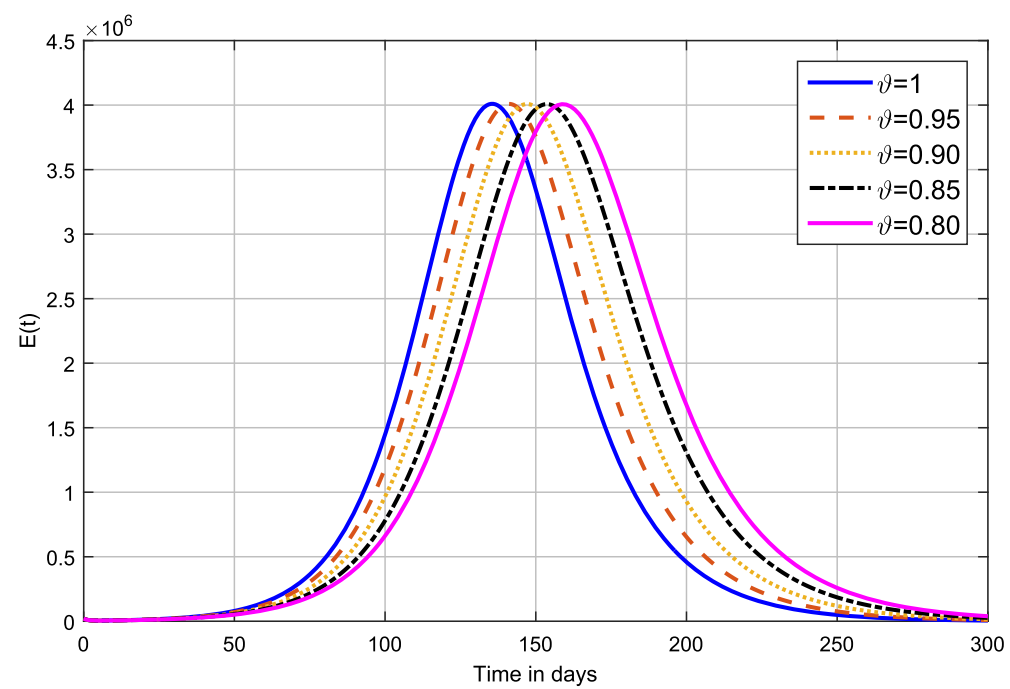

Fig. 3 Dynamics of exposed individuals for various values of $\vartheta$

proceed as follows:

$$
\mathcal{G}(x, \mathrm{Y}(x)) \cong \mathcal{P}_{k}(x)=\frac{\mathrm{G}\left(t_{l}, \mathrm{Y}\left(t_{l}\right)\right)}{h}\left(x-t_{l-1}\right)-\frac{\mathrm{G}\left(t_{l-1}, \mathrm{Y}\left(t_{l-1}\right)\right)}{h}\left(x-t_{l}\right)
$$




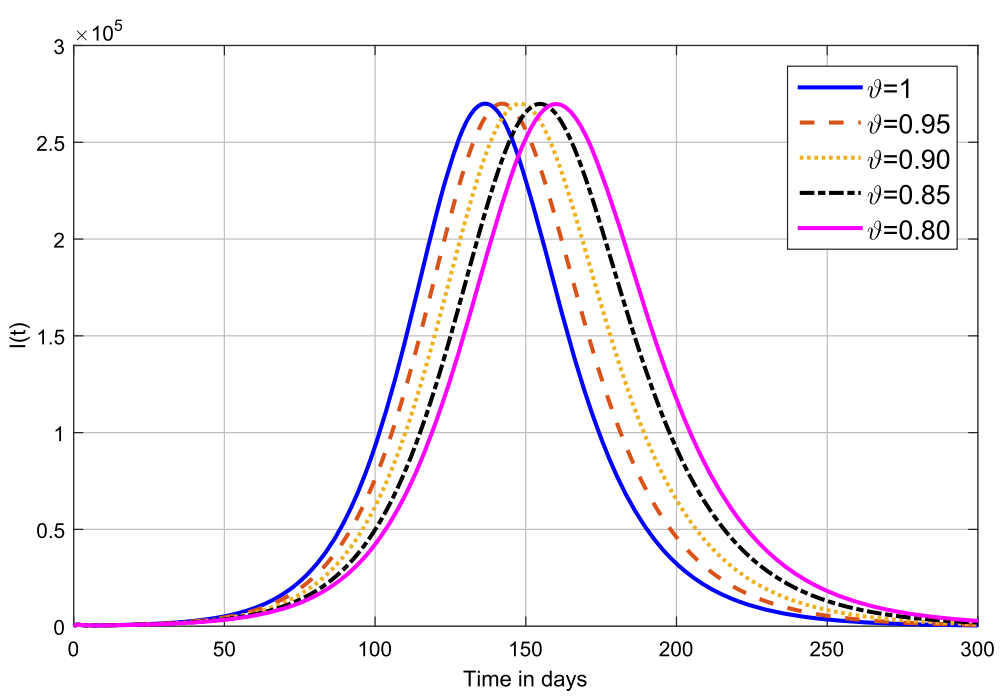

Fig. 4 Dynamics of symptomatic individuals for various values of $\vartheta$

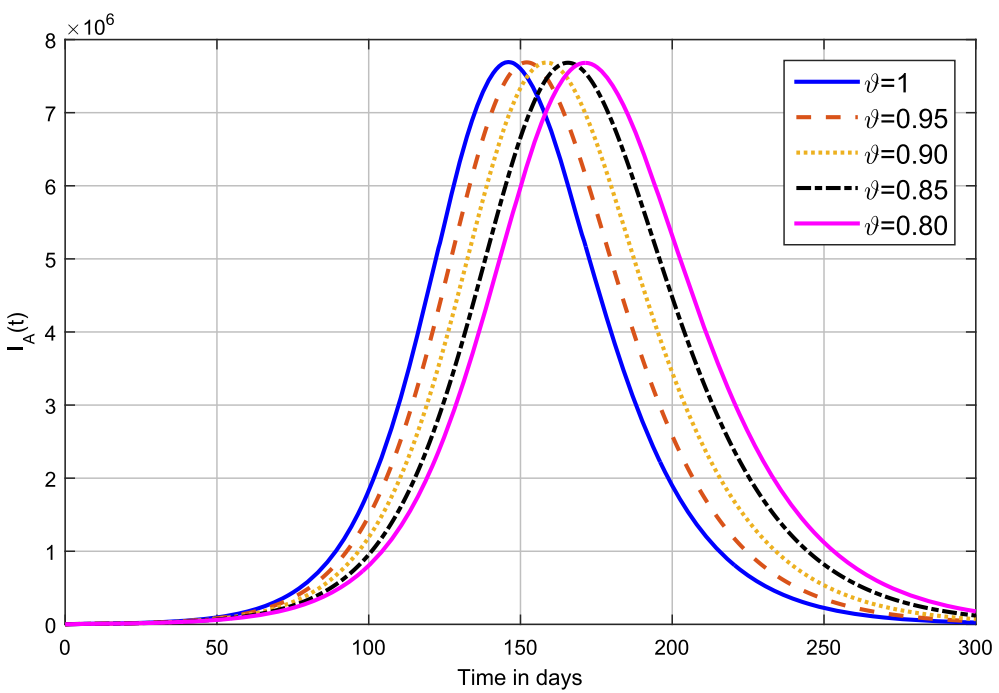

Fig. 5 Dynamics of asymptomatic individuals for various values of $\vartheta$

So, Eq. (24) becomes:

$$
\begin{aligned}
\mathrm{y}\left(t_{\mathrm{k}+1}\right)= & \mathrm{y}(0)+\frac{1-\vartheta}{A B(\vartheta)} \mathrm{G}\left(t_{\mathrm{k}}, \mathrm{Y}\left(t_{\mathrm{k}}\right)\right) \\
& +\frac{\vartheta}{A B(\vartheta) \times \Gamma(\vartheta)} \sum_{l=0}^{\mathrm{k}}\left(\frac{\mathrm{G}\left(t_{l}, \mathrm{y}\left(t_{l}\right)\right)}{h} \int_{t_{l}}^{t_{l+1}}\left(x-t_{l-1}\right)\left(t_{\mathrm{k}+1}-x\right)^{\vartheta-1} \mathrm{~d} x\right. \\
& \left.-\frac{\mathrm{G}\left(t_{l-1}, \mathrm{y}\left(t_{l-1}\right)\right)}{h} \int_{t_{l}}^{t_{l+1}}\left(x-t_{l}\right)\left(t_{\mathrm{k}+1}-x\right)^{\vartheta-1} \mathrm{~d} x\right) .
\end{aligned}
$$




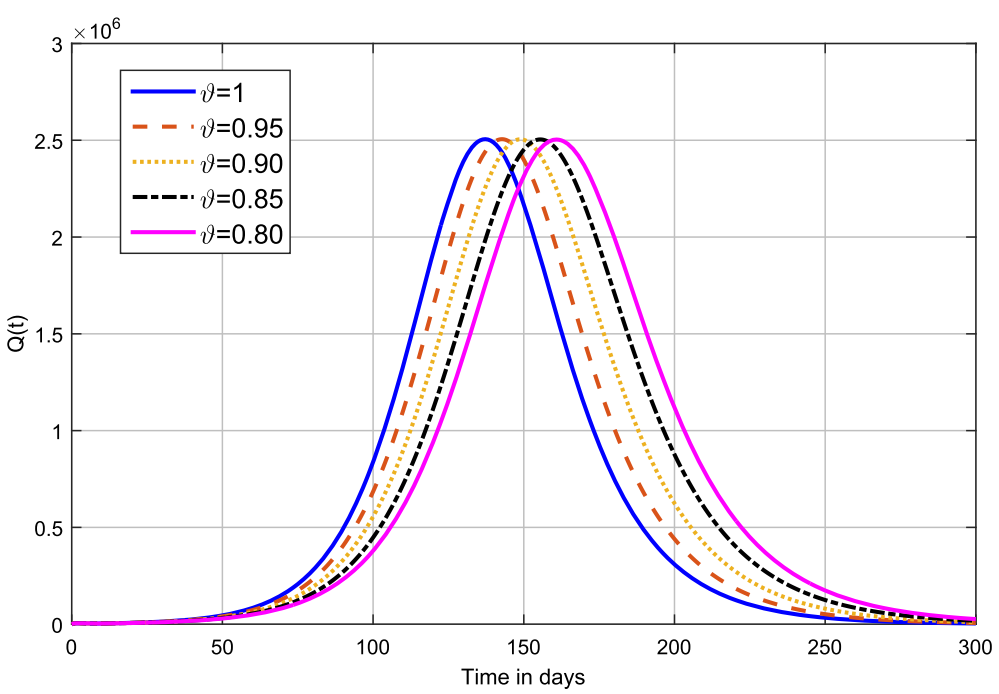

Fig. 6 Dynamics of quarantine individuals for various values of $\vartheta$

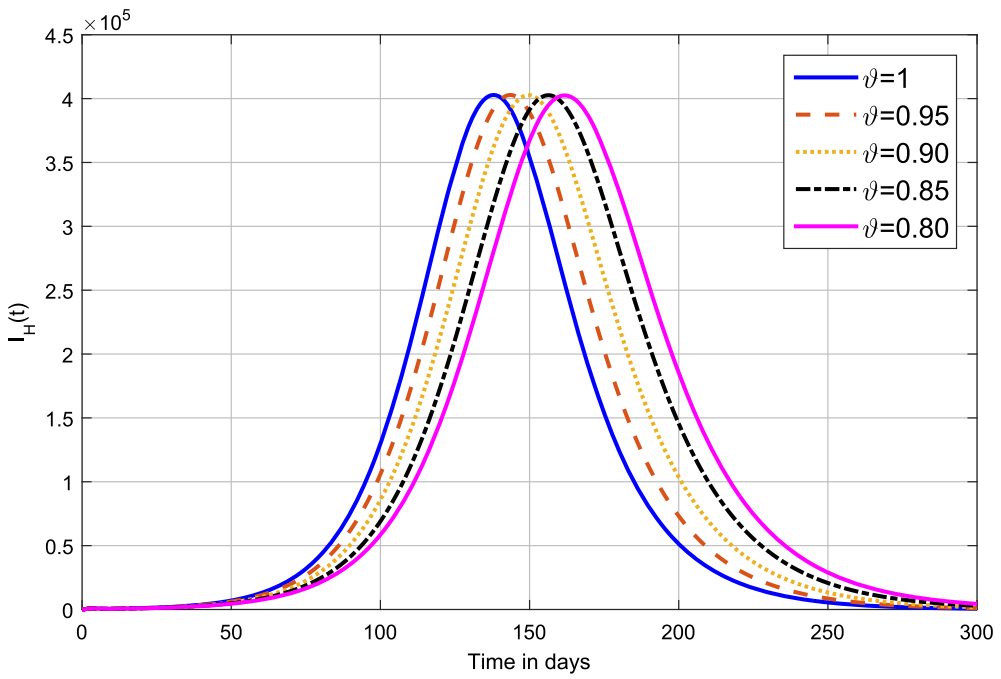

Fig. 7 Dynamics of hospitalized or self-isolated individuals for various values of $\vartheta$

On further simplification of the integrals in (26), the approximate solution for the problem in consideration is as follows:

$$
\begin{aligned}
\mathrm{y}\left(t_{\mathrm{k}+1}\right)= & \mathrm{y}\left(t_{0}\right)+\frac{1-\vartheta}{A B(\vartheta)} \mathrm{G}\left(t_{\mathrm{k}}, \mathrm{y}\left(t_{\mathrm{k}}\right)\right)+\frac{\vartheta}{A B(\vartheta)} \sum_{l=0}^{\mathrm{k}} \\
& \left(\frac{h^{\vartheta} \mathrm{G}\left(t_{l}, \mathrm{Y}\left(t_{l}\right)\right)}{\Gamma(\vartheta+2)}\left\{(\mathrm{k}-l+1)^{\vartheta}(\mathrm{k}-l+2+\vartheta)-(\mathrm{k}-l)^{\vartheta}(\mathrm{k}-l+2+2 \vartheta)\right\}\right. \\
& \left.-\frac{h^{\vartheta} \mathrm{G}\left(t_{l-1}, \mathrm{y}\left(t_{l-1}\right)\right)}{\Gamma(\vartheta+2)}\left\{(\mathrm{k}-l+1)^{\vartheta+1}-(\mathrm{k}-l)^{\vartheta}(\mathrm{k}-l+1+\vartheta)\right\}\right) .
\end{aligned}
$$




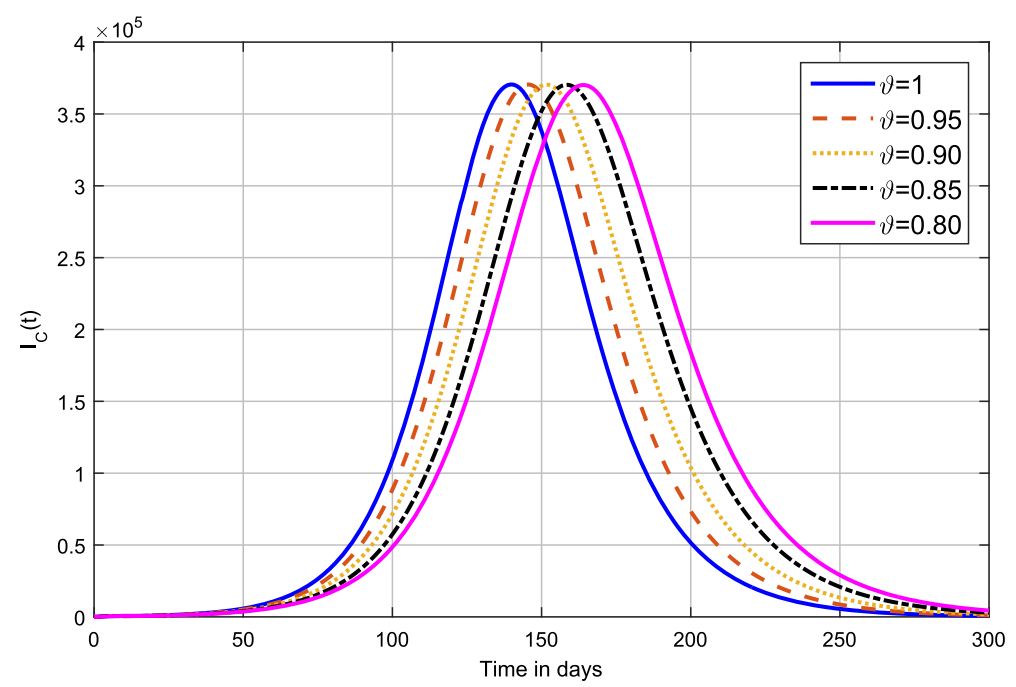

Fig. 8 Dynamics of critically infected individuals for various values of $\vartheta$

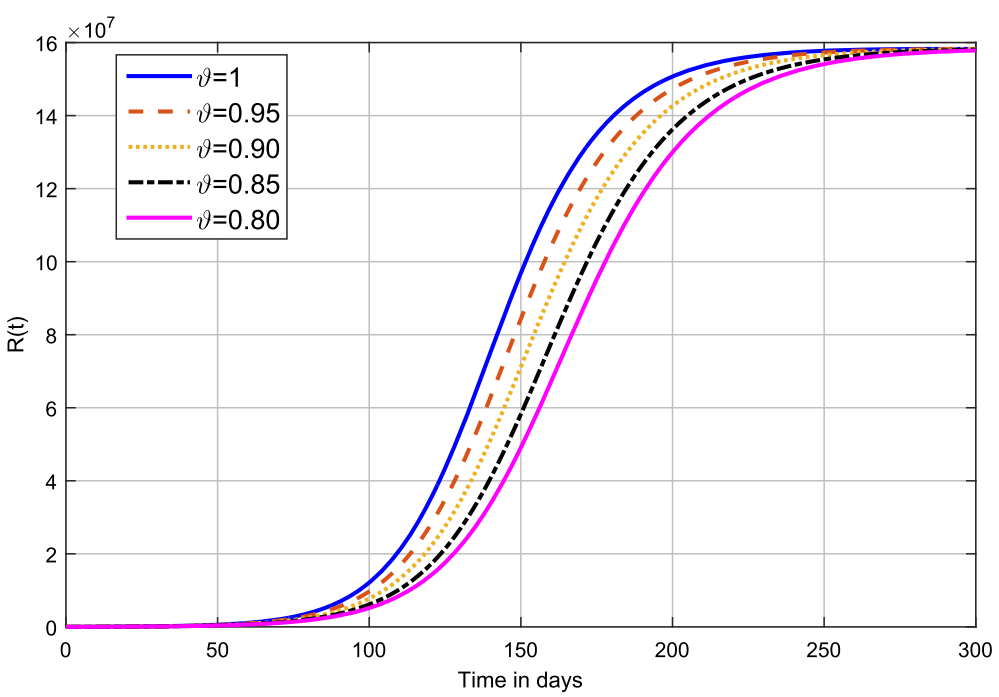

Fig. 9 Dynamics of recovered individuals for various values of $\vartheta$

Thus, the following recursive expressions are obtained for the ABC fractional COVID-19 epidemic model described in (8):

$$
\begin{aligned}
S\left(t_{\mathrm{k}+1}\right)= & S\left(t_{0}\right)+\frac{1-\vartheta}{A B(\vartheta)} \mathrm{G}_{1}\left(t_{\mathrm{k}}, \mathrm{y}\left(t_{\mathrm{k}}\right)\right)+\frac{\vartheta}{A B(\vartheta)} \sum_{l=0}^{\mathrm{k}} \\
& \left(\frac{h^{\vartheta} \mathrm{G}_{1}\left(t_{l}, \mathrm{y}\left(t_{l}\right)\right)}{\Gamma(\vartheta+2)}\left\{(\mathrm{k}+1-l)^{\vartheta}(\mathrm{k}+2-l+\vartheta)-(\mathrm{k}-l)^{\vartheta}(\mathrm{k}+2-l+2 \vartheta)\right\}\right. \\
& \left.-\frac{h^{\vartheta} \mathrm{G}_{1}\left(t_{l-1}, \mathrm{y}\left(t_{l-1}\right)\right)}{\Gamma(\vartheta+2)}\left\{(\mathrm{k}-l+1)^{\vartheta+1}-(\mathrm{k}-l)^{\vartheta}(\mathrm{k}-l+1+\vartheta)\right\}\right),
\end{aligned}
$$



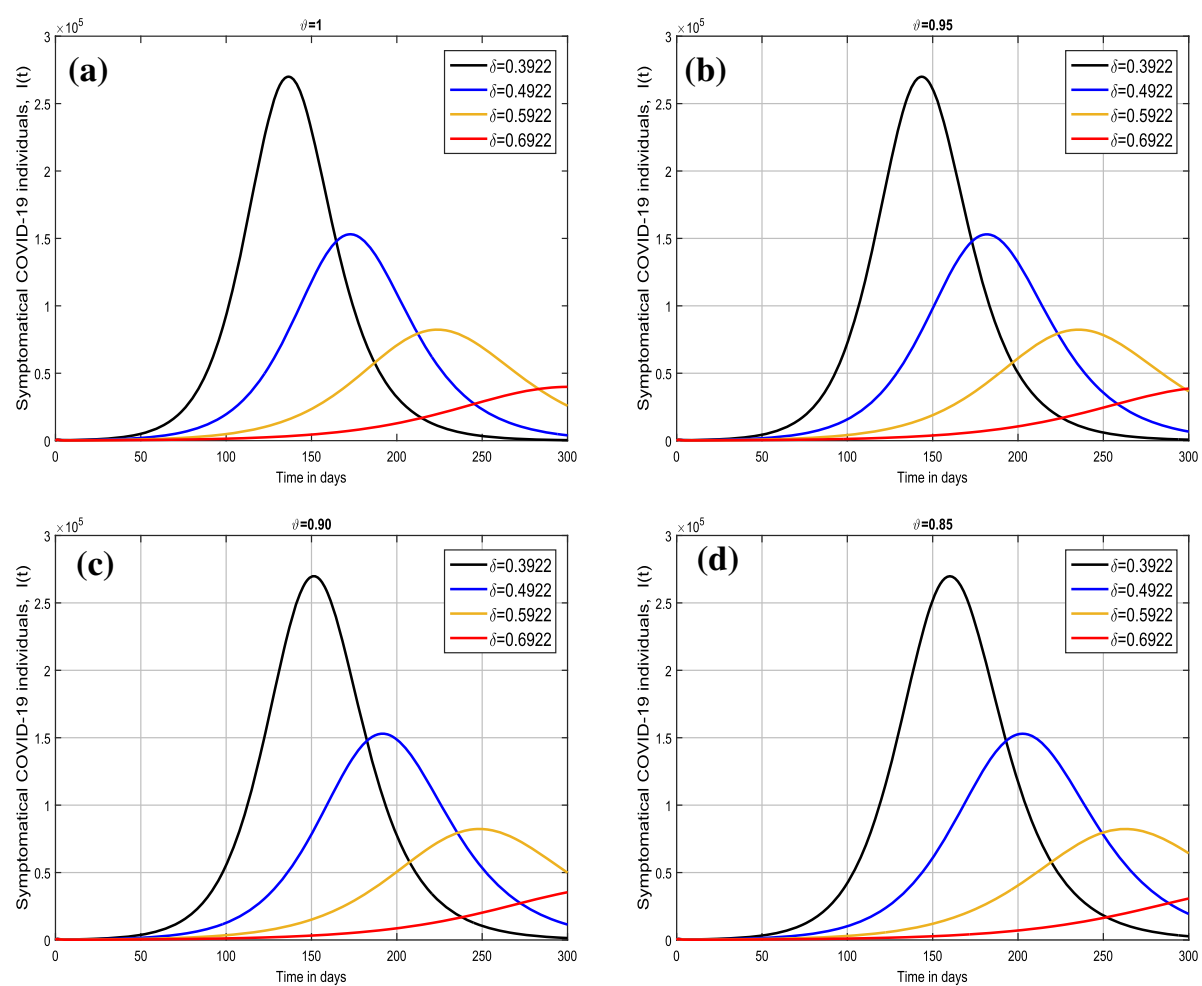

Fig. 10 The effect of $\delta$ (quarantine rate) on the symptomatically infected people for $\mathbf{a} \vartheta=1$, b $\vartheta=0.95$, c $\vartheta=0.90, \mathbf{d} \vartheta=0.85$

$$
\begin{aligned}
E\left(t_{\mathrm{k}+1}\right)= & E\left(t_{0}\right)+\frac{1-\vartheta}{A B(\vartheta)} \mathrm{G}_{2}\left(t_{\mathrm{k}}, \mathrm{y}\left(t_{\mathrm{k}}\right)\right)+\frac{\vartheta}{A B(\vartheta)} \sum_{l=0}^{\mathrm{k}} \\
& \left(\frac{h^{\vartheta} \mathrm{G}_{2}\left(t_{l}, \mathrm{y}\left(t_{l}\right)\right)}{\Gamma(\vartheta+2)}\left\{(\mathrm{k}+1-l)^{\vartheta}(\mathrm{k}+2-l+\vartheta)-(\mathrm{k}-l)^{\vartheta}(\mathrm{k}+2-l+2 \vartheta)\right\}\right. \\
& \left.-\frac{h^{\vartheta} \mathrm{G}_{2}\left(t_{l-1}, \mathrm{y}\left(t_{l-1}\right)\right)}{\Gamma(\vartheta+2)}\left\{(\mathrm{k}+1-l)^{\vartheta+1}-(\mathrm{k}-l)^{\vartheta}(\mathrm{k}-l+1+\vartheta)\right\}\right), \\
I\left(t_{\mathrm{k}+1}\right)= & I\left(t_{0}\right)+\frac{1-\vartheta}{B(\vartheta)} \mathrm{G}_{3}\left(t_{\mathrm{k}}, \mathrm{y}\left(t_{\mathrm{k}}\right)\right)+\frac{\vartheta}{A B(\vartheta)} \sum_{l=0}^{\mathrm{k}} \\
& \left(\frac{h^{\vartheta} \mathrm{G}_{3}\left(t_{l}, \mathrm{y}\left(t_{l}\right)\right)}{\Gamma(\vartheta+2)}\left\{(\mathrm{k}+1-l)^{\vartheta}(\mathrm{k}+2-l+\vartheta)-(\mathrm{k}-l)^{\vartheta}(\mathrm{k}+2-l+2 \vartheta)\right\}\right. \\
& \left.-\frac{h^{\vartheta} \mathrm{G}_{3}\left(t_{l-1}, \mathrm{y}\left(t_{l-1}\right)\right)}{\Gamma(\vartheta+2)}\left\{(\mathrm{k}+1-l)^{\vartheta+1}-(\mathrm{k}-l)^{\vartheta}(\mathrm{k}-l+1+\vartheta)\right\}\right), \\
& \left(\frac{h^{\vartheta} \mathrm{G}_{4}\left(t_{l}, \mathrm{y}\left(t_{l}\right)\right)}{\Gamma(\vartheta+2)}\left\{(\mathrm{k}+1-l)^{\vartheta}(\mathrm{k}+2-l+\vartheta)-(\mathrm{k}-l)^{\vartheta}(\mathrm{k}+2-l+2 \vartheta)\right\}\right. \\
I_{A}\left(t_{\mathrm{k}+1}\right)= & I_{A}\left(t_{0}\right)+\frac{1-\vartheta}{A B(\vartheta)} \mathrm{G}_{4}\left(t_{\mathrm{k}}, \mathrm{y}\left(t_{\mathrm{k}}\right)\right)+\frac{\vartheta}{A B(\vartheta)} \sum_{l=0}^{\mathrm{k}}
\end{aligned}
$$



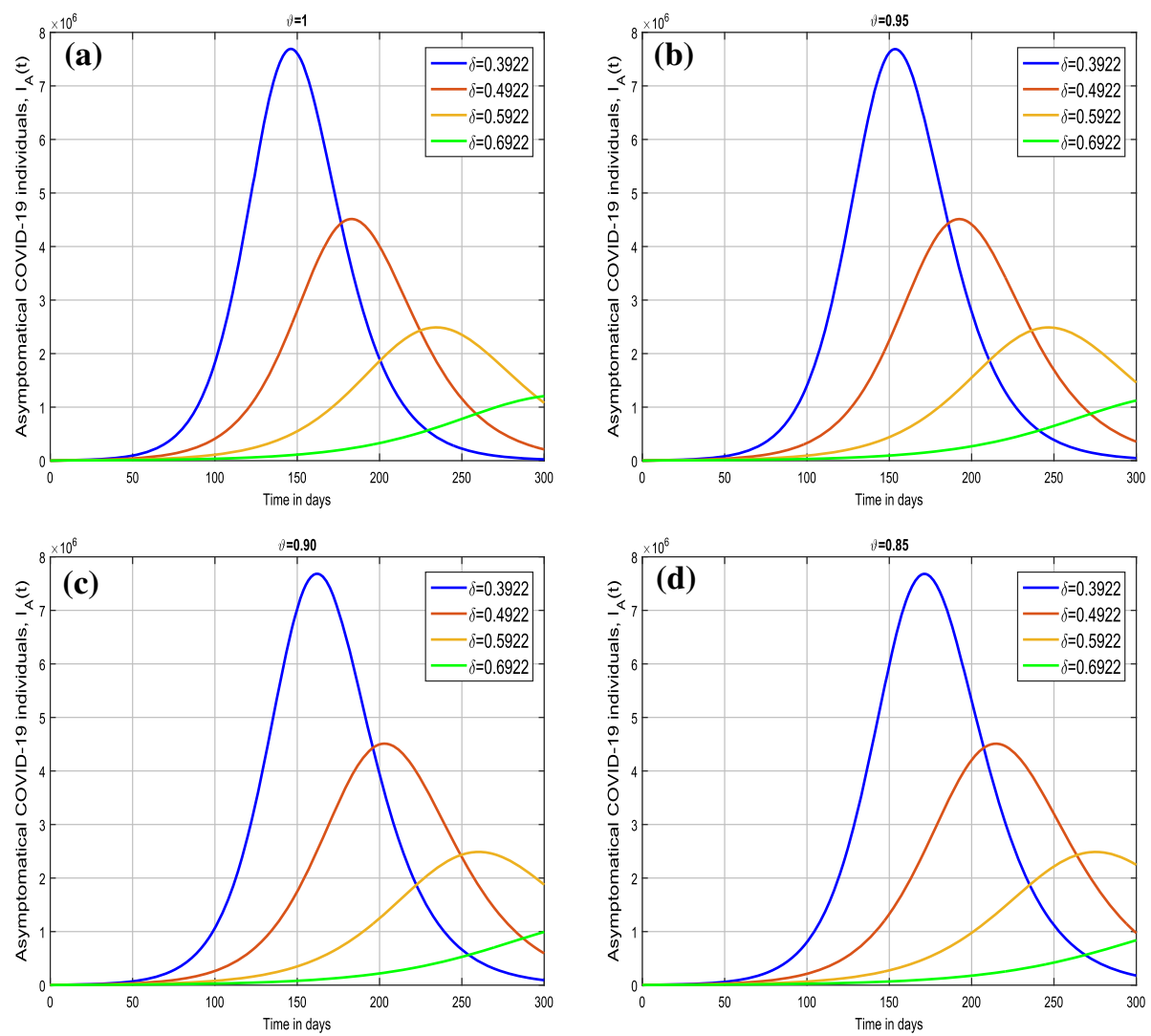

Fig. 11 The effect $\delta$ (quarantine rate) on the asymptomatically infected people for a $\vartheta=1, \mathbf{b} \vartheta=0.95$, c $\vartheta=0.90, \mathbf{d} \vartheta=0.85$

$$
\begin{aligned}
& \left.-\frac{h^{\vartheta} \mathrm{G}_{4}\left(t_{l-1}, \mathrm{y}\left(t_{l-1}\right)\right)}{\Gamma(\vartheta+2)}\left\{(\mathrm{k}+1-l)^{\vartheta+1}-(\mathrm{k}-l)^{\vartheta}(\mathrm{k}-l+1+\vartheta)\right\}\right), \\
Q\left(t_{\mathrm{k}+1}\right)= & Q\left(t_{0}\right)+\frac{1-\vartheta}{A B(\vartheta)} \mathrm{G}_{5}\left(t_{\mathrm{k}}, \mathrm{y}\left(t_{\mathrm{k}}\right)\right)+\frac{\vartheta}{A B(\vartheta)} \sum_{l=0}^{\mathrm{k}} \\
& \left(\frac{h^{\vartheta} \mathrm{G}_{5}\left(t_{l}, \mathrm{y}\left(t_{l}\right)\right)}{\Gamma(\vartheta+2)}\left\{(\mathrm{k}+1-l)^{\vartheta}(\mathrm{k}+2-l+\vartheta)-(\mathrm{k}-l)^{\vartheta}(\mathrm{k}+2-l+2 \vartheta)\right\}\right. \\
& \left.-\frac{h^{\vartheta} \mathrm{G}_{5}\left(t_{l-1}, \mathrm{y}\left(t_{l-1}\right)\right)}{\Gamma(\vartheta+2)}\left\{(\mathrm{k}+1-l)^{\vartheta+1}-(\mathrm{k}-l)^{\vartheta}(\mathrm{k}-l+1+\vartheta)\right\}\right), \\
I_{H}\left(t_{\mathrm{k}+1}\right)= & I_{H}\left(t_{0}\right)+\frac{1-\vartheta}{A B(\vartheta)} \mathrm{G}_{6}\left(t_{\mathrm{k}}, \mathrm{y}\left(t_{\mathrm{k}}\right)\right)+\frac{\vartheta}{A B(\vartheta)} \sum_{l=0}^{\mathrm{k}} \\
& \left(\frac{h^{\vartheta} \mathrm{G}_{6}\left(t_{l}, \mathrm{y}\left(t_{l}\right)\right)}{\Gamma(\vartheta+2)}\left\{(\mathrm{k}+1-l)^{\vartheta}(\mathrm{k}+2-l+\vartheta)-(\mathrm{k}-l)^{\vartheta}(\mathrm{k}+2-l+2 \vartheta)\right\}\right. \\
& \left.-\frac{h^{\vartheta} \mathrm{G}_{6}\left(t_{l-1}, \mathrm{y}\left(t_{l-1}\right)\right)}{\Gamma(\vartheta+2)}\left\{(\mathrm{k}+1-l)^{\vartheta+1}-(\mathrm{k}-l)^{\vartheta}(\mathrm{k}-l+1+\vartheta)\right\}\right),
\end{aligned}
$$




$$
\begin{aligned}
I_{C}\left(t_{\mathrm{k}+1}\right)= & I_{C}\left(t_{0}\right)+\frac{1-\vartheta}{A B(\vartheta)} \mathrm{G}_{7}\left(t_{\mathrm{k}}, \mathrm{y}\left(t_{\mathrm{k}}\right)\right)+\frac{\vartheta}{A B(\vartheta)} \sum_{l=0}^{\mathrm{k}} \\
& \left(\frac{h^{\vartheta} \mathrm{G}_{7}\left(t_{l}, \mathrm{y}\left(t_{l}\right)\right)}{\Gamma(\vartheta+2)}\left\{(\mathrm{k}+1-l)^{\vartheta}(\mathrm{k}+2-l+\vartheta)-(\mathrm{k}-l)^{\vartheta}(\mathrm{k}+2-l+2 \vartheta)\right\}\right. \\
& \left.-\frac{h^{\vartheta} \mathrm{G}_{7}\left(t_{l-1}, \mathrm{y}\left(t_{l-1}\right)\right)}{\Gamma(\vartheta+2)}\left\{(\mathrm{k}+1-l)^{\vartheta+1}-(\mathrm{k}-l)^{\vartheta}(\mathrm{k}-l+1+\vartheta)\right\}\right), \\
R\left(t_{\mathrm{k}+1}\right)= & R\left(t_{0}\right)+\frac{1-\vartheta}{A B(\vartheta)} \mathrm{G}_{8}\left(t_{\mathrm{k}}, \mathrm{y}\left(t_{\mathrm{k}}\right)\right)+\frac{\vartheta}{A B(\vartheta)} \sum_{l=0}^{\mathrm{k}} \\
& \left(\frac{h^{\vartheta} \mathrm{G}_{8}\left(t_{l}, \mathrm{y}\left(t_{l}\right)\right)}{\Gamma(\vartheta+2)}\left\{(\mathrm{k}+1-l)^{\vartheta}(\mathrm{k}+2-l+\vartheta)-(\mathrm{k}-l)^{\vartheta}(\mathrm{k}+2-l+2 \vartheta)\right\}\right. \\
& \left.-\frac{h^{\vartheta} \mathrm{G}_{8}\left(t_{l-1}, \mathrm{y}\left(t_{l-1}\right)\right)}{\Gamma(\vartheta+2)}\left\{(\mathrm{k}+1-l)^{\vartheta+1}-(\mathrm{k}-l)^{\vartheta}(\mathrm{k}-l+1+\vartheta)\right\}\right) . \quad(28)
\end{aligned}
$$

\section{Numerical results and discussion}

In this section, we present the simulation results of the fractional COVID-19 epidemic model in the $\mathrm{ABC}$ sense (8). For this purpose, we utilize the iterative scheme developed in the previous section and values of the parameters are given in Table 1. The time level taken into consideration in simulations is 300 days. The initial conditions are taken from [13]. Initially, we demonstrate the impact of memory index (the order of $\mathrm{ABC}$ operator $\vartheta$ ) on the dynamics of different population classes in the model (8). The dynamical behavior of susceptible individuals is described in Fig. 2. We observed that for smaller values $\vartheta$ the susceptible population initially increased until becoming stable after 250 days. Similarly, the influence of various values of the fractional order $\vartheta$ on the dynamics of exposed, symptomatic, asymptomatic infected people and quarantine, hospitalized and critically infected COVID19 individuals is described in Figs. 3, 4, 5, 6, 7 and 8, respectively. The same behavior is observed for all population compartments as can be seen in 3, 4, 5, 6, 7 and 8 . The simulation results describing the dynamics of the recovered individuals for different memory index $\vartheta$ are shown in Fig. 9. The recovered population decreases for smaller values of $\vartheta$ and then finally became stable after 250 days. Moreover, to analyze the impacts of quarantine rate $\delta$ on the dynamics of cumulative symptomatic and asymptomatic COVID-19 infective individuals we vary this parameter with different rates and depict the simulations. The resulting graphical interpretations are carried out for four values of $\vartheta$. From Fig. 10, one can observe that the symptomatic infected population is decreased significantly as we increase the quarantine rate $\delta$ to its baseline value. This interpretation is carried out for different values of $\vartheta$ as can be seen in Fig. 10(a)-(d). Finally, the importance of variation in $\delta$ is analyzed in Fig. 11.

\section{Conclusion}

The epidemic models formulated using the non-integer-order operators provide comparatively deeper insights into the disease transmission dynamics. In addition, the fractional derivative based on generalized Mittag-Leffler-type kernel is capable of capturing the crossover features found in the various biological problems. In this study, we explored the 
transmission dynamics of the novel COVID-19 pandemic using a fractional-order mathematical model in Atangana-Baleanu-Caputo sense. Initially, we briefly reviewed the classical integer-order mathematical model for the COVID-19 dynamics. The classical model is then extended to fractional environment with the help of $\mathrm{ABC}$ operator. In order to obtain the fractional model, we replaced the classical derivative with the $\mathrm{ABC}$ operator in the model. Some of the basic mathematical properties of the ABC COVID-19 epidemic model are carried out. An efficient modified Adams-Bashforth is applied to obtain an iterative scheme of the proposed fractional model. Moreover, detailed simulation results of the ABC mathematical model are presented to demonstrate the importance of the memory index $\vartheta$ and model parameters on the transmission and control of COVID-19 infection. Thus, from the interpretation presented in this study, we conclude that the models constructed in non-integer orders exhibit many characteristics that cannot be observed in integer order and more interesting results can be obtained. The studied will be extended in the near future to obtain its solutions using other operators in fractional derivatives.

\section{References}

1. E. Ortiz-Ospina, M. Roser, H. Ritchie and J. Hasell, Coronavirus pandemic (covid-19). Our World in Data. (2020). https://ourworldindata.org/coronavirus

2. Pakistan Population (1950-2020). https://www.worldometers.info/world-population/pakistanpopulation/

3. World Health Organization, https://www.who.int/emergencies/diseases/novel-coronavirus-2019. Accessed 30th June 2020

4. Center for Disease Control and Prevention (CDC). https://www.cdc.gov/coronavirus/2019-ncov/index. html

5. A. Yusuf, S. Qureshi, M. Inc, A.I. Aliyu, D. Baleanu, A.A. Shaikh, Two-strain epidemic model involving fractional derivative with Mittag-Leffler kernel. Chaos Interdiscip. J. Nonlinear Sci. 28(12), 123121 (2018)

6. A. Jajarmi, A. Yusuf, D. Baleanu, M. Inc, A new fractional HRSV model and its optimal control: a non-singular operator approach. Phys. A Stat. Mech. Appl. 547, 123860 (2020)

7. M. Inc, A. Yusuf, A.I. Aliyu, D. Baleanu, Time-fractional Cahn-Allen and time-fractional Klein-Gordon equations: Lie symmetry analysis, explicit solutions and convergence analysis. Phys. A Stat. Mech. Appl. 493, 94-106 (2018)

8. A.I. Aliyu, M. Inc, A. Yusuf, D. Baleanu, A fractional model of vertical transmission and cure of vectorborne diseases pertaining to the Atangana-Baleanu fractional derivatives. Chaos Solitons Fractals 116, 268-277 (2018)

9. M. Inc, A. Yusuf, A.I. Aliyu, D. Baleanu, Lie symmetry analysis, explicit solutions and conservation laws for the space-time fractional nonlinear evolution equations. Phys. A Stat. Mech. Appl. 496, 371-383 (2018)

10. S. Qureshi, A. Yusuf, A.A. Shaikh, M. Inc, Transmission dynamics of varicella zoster virus modeled by classical and novel fractional operators using real statistical data. Phys. A Stat. Mech. Appl. 534, 122149 (2019)

11. A. Khan, R. Zarin, G. Zaman, B. Almohsen et al., Stability analysis of leishmania epidemic model with harmonic mean type incidence rate. Eur. Phys. J. Plus 135(6), 1-20 (2020)

12. A. Khan, G. Hussain, M. Inc, G. Zaman, Existence, uniqueness, and stability of fractional hepatitis B epidemic model. Chaos Interdiscip. J. Nonlinear Sci. 30(10), 103104 (2020)

13. S. Ullah, M.A. Khan, Modeling the impact of non-pharmaceutical interventions on the dynamics of novel coronavirus with optimal control analysis with a case study. Chaos Solitons Fractals 139, 110075 (2020)

14. M.S. Alqarni, M. Alghamdi, T. Muhammad, A.S. Alshomrani, and M.A. Khan, Mathematical modeling for novel coronavirus (COVID-19) and control. Numer. Methods Partial Differ. Equ. (2020)

15. J.K.K. Asamoah, M.A. Owusu, Z. Jin, F.T. Oduro, A. Abidemi, E.O. Gyasi, Global stability and costeffectiveness analysis of COVID-19 considering the impact of the environment: using data from Ghana. Chaos Solitons Fractals 140, 110103 (2020)

16. S. Annas, M.I. Pratama, M. Rifandi, W. Sanusi, S. Side, Stability analysis and numerical simulation of SEIR model for pandemic COVID-19 spread in Indonesia. Chaos Solitons Fractals 139, 110072 (2020) 
17. S. Kumar, R.P. Chauhan, S. Momani, S. Hadid, Numerical investigations on COVID-19 model through singular and non-singular fractional operators. Numer. Methods Partial Differ. Equ. (2020). https://doi. org/10.1002/num. 22707

18. K.M. Safare, D.G. ViS Betageri, P.V. Prakasha, S. Kumar, A mathematical analysis of ongoing outbreak COVID-19 in India through nonsingular derivative. Numer. Methods Partial Differ. Equ. (2020). https:// doi.org/10.1002/num.22579

19. S. Ullah, M.A. Khan, M. Farooq, A fractional model for the dynamics of TB virus. Chaos Solitons Fractals 116, 63-71 (2018)

20. P.A. Naik, M. Yavuz, S. Qureshi, Z. Jian, S. Townley, Modeling and analysis of COVID-19 epidemics with treatment in fractional derivatives using real data from Pakistan. Eur. Phys. J. Plus 135(10), 1-42 (2020)

21. A. Atangana, and D. Baleanu, New fractional derivatives with nonlocal and non-singular kernel: theory and application to heat transfer model. arXiv preprint arXiv:1602.03408 (2016)

22. I. Podlubny, Fractional Differential Equations: An Introduction to Fractional Derivatives, Fractional Differential Equations, to Methods of Their Solution and Some of Their Applications (Elsevier, Amsterdam, 1998)

23. M.A. Khan, A. Atangana, Modeling the dynamics of novel coronavirus (2019-nCov) with fractional derivative. Alex. Eng. J. 59, 2379-2389 (2020)

24. D. Baleanu, H. Mohammadi, S. Rezapour, A fractional differential equation model for the COVID-19 transmission by using the Caputo-Fabrizio derivative. Adv. Differ. Equ. 2020(1), 1-27 (2020)

25. N.H. Tuan, H. Mohammadi, S. Rezapour, A mathematical model for COVID-19 transmission by using the Caputo fractional derivative. Chaos Solitons Fractals 140, 110107 (2020)

26. M. Awais, F.S. Alshammari, S. Ullah, M.A. Khan, S. Islam, Modeling and simulation of the novel coronavirus in Caputo derivative. Results Phys. 19, 103588 (2020)

27. A. Ali, F.S. Alshammari, S. Islam, M.A. Khan, S. Ullah, Modeling and analysis of the dynamics of novel coronavirus (COVID-19) with Caputo fractional derivative. Results Phys. 20, 103669 (2020)

28. Y.-M. Chu, A. Ali, M.A. Khan, S. Islam, and S. Ullah, Dynamics of fractional order COVID-19 model with a case study of Saudi Arabia. Results Phys. p. 103787 (2021)

29. M.A. Taneco-Hernández, C. Vargas-De-Leon, Stability and Lyapunov functions for systems with Atangana-Baleanu Caputo derivative: an HIV/AIDS epidemic model. Chaos Solitons Fractals 132, $109586(2020)$

30. P. Van den Driessche, J. Watmough, Reproduction numbers and sub-threshold endemic equilibria for compartmental models of disease transmission. Math. Biosci. 180(1-2), 29-48 (2002)

31. M. Toufik, A. Atangana, New numerical approximation of fractional derivative with non-local and nonsingular kernel: application to chaotic models. Eur. Phys. J. Plus 132(10), 444 (2017) 